\title{
Establishment of caprine airway epithelial cells grown in an air-liquid interface system to study caprine respiratory viruses and bacteria
}

\author{
Marina Strässle $^{\text {a,b,c }}$, Laura Laloli ${ }^{\text {a,c,d,g }}$, Mitra Gultom ${ }^{\text {a,c,d,g }}$, Philip V'kovski ${ }^{\text {a,c }}$, \\ Michael H. Stoffel ${ }^{\mathrm{e}}$, Silvia Crespo Pomar ${ }^{\mathrm{b}}$, Astrid Chanfon Bätzner ${ }^{\mathrm{f}}$, Nadine Ebert ${ }^{\mathrm{a}, \mathrm{c}}$, \\ Fabien Labroussaa $^{\mathrm{b}}$, Ronald Dijkman ${ }^{\mathrm{a}, \mathrm{c}, \mathrm{g}}$, Joerg Jores ${ }^{\mathrm{b},{ }^{\prime}, 1}$, Volker Thiel ${ }^{\mathrm{a}, \mathrm{c}, 1}$

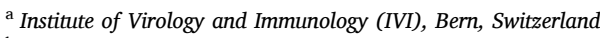 \\ ${ }^{\mathrm{b}}$ Institute of Veterinary Bacteriology, Department of Infectious Diseases and Pathobiology, Vetsuisse Faculty, University of Bern, Länggass-Str. 122, PO Box 3001, Bern, \\ Switzerland \\ ${ }^{\mathrm{c}}$ Department of Infectious Diseases and Pathobiology, Vetsuisse Faculty, University of Bern, Bern, Switzerland \\ ${ }^{\mathrm{d}}$ Graduate School for Biomedical Science, University of Bern, Bern, Switzerland \\ ${ }^{\mathrm{e}}$ Division of Veterinary Anatomy, Vetsuisse Faculty, University of Bern, Länggass-Str. 120, PO Box 3001, Bern, Switzerland \\ ${ }^{\mathrm{f}}$ Institute of Animal Pathology (COMPATH), Vetsuisse Faculty, University of Bern, Länggass-Str. 122, PO Box 3001, Bern, Switzerland \\ ${ }^{g}$ Institute for Infectious Diseases, University of Bern, Bern, Switzerland
}

\section{A R T I C L E I N F O}

\section{Keywords:}

Airway epithelial cell culture

AECs

Goat

Air liquid interface

ALI

Influenza D virus

Mycoplasma mycoides subsp. capri

\begin{abstract}
A B S T R A C T
Respiratory diseases negatively impact the global goat industry, but are understudied. There is a shortage of established and biological relevant in vitro or ex vivo assays to study caprine respiratory infections. Here, we describe the establishment of an in vitro system based on well-differentiated caprine airway epithelial cell (AEC) cultures grown under air liquid interface conditions as an experimental platform to study caprine respiratory pathogens. The functional differentiation of the AEC cultures was monitored and confirmed by light and immunofluorescence microscopy, scanning electron microscopy and examination of histological sections. We validated the functionality of the platform by studying Influenza D Virus (IDV) infection and Mycoplasma mycoides subsp. capri $(\mathrm{Mmc})$ colonization over 5 days, including monitoring of infectious agents by titration and qPCR as well as colour changing units, respectively. The inoculation of caprine AEC cultures with IDV showed that efficient viral replication takes place, and revealed that IDV has a marked cell tropism for ciliated cells. Furthermore, AEC cultures were successfully infected with $M m c$ using a multiplicity of infection of 0.1 and colonization was monitored over several days. Altogether, these results demonstrate that our newly-established caprine AEC cultures can be used to investigate host-pathogen interactions of caprine respiratory pathogens.
\end{abstract}

\section{Introduction}

Goats are an important asset for livestock-dependent communities, especially in low- and middle-income countries in Africa and Asia. Over the last decades, the global goat industry, especially the dairy goat husbandry, has expanded rapidly (Miller and Lu, 2019). The global goat population is estimated to be more than 1 billion goats (FAO, 2020) with Asian countries and specifically China accounting for more than $50 \%$ of it. Infectious diseases hamper the productivity and animal welfare of goats. Specifically, respiratory infections, such as contagious caprine pleuropneumonia (Jores et al., 2020) or peste des petits ruminants
(Banyard et al., 2010), are a leading cause of low productivity and mortality in goats (Chakraborty et al., 2014). Investigation of host-pathogen interactions relies on in vitro, ex vivo and in vivo assays, whereby the latter are most informative but have serious animal welfare and cost implication. Therefore, the availability of in vitro and ex vitro assay surrogates of caprine lung tissue fosters the investigation of caprine respiratory pathogens. In vitro assays based on immortalized or primary cells derived from the caprine respiratory tissue have contributed greatly to our understanding of host-pathogen interactions such as the investigation of host specificity of different respiratory mycoplasmas (Aye et al., 2015) and Influenza viruses (Rosenberger et al., 2014).

\footnotetext{
* Corresponding author.

E-mail address: joerg.jores@vetsuisse.unibe.ch (J. Jores).

${ }^{1}$ Contributed equally
} 
However, these cell lines when submerged in cell culture medium do not mimic the complex cellular organization and cell diversity of the respiratory tissue. Recently, a precision-cut lung slices (PCLS) assay for goats has been established and used to investigate adhesion of caprine pathogenic mycoplasmas (Weldearegay et al., 2019). PCLS reflect the lung tissue adequately but require a constant source of clean donor animals and do not contain a distinct air compartment, which is characteristic for respiratory tissue.

Here we developed a novel in vitro platform to study caprine respiratory infections. This platform is based on the generation of caprine primary airway epithelial cell (AEC) cultures that are welldifferentiated, grown under so-called "air-liquid-interface" (ALI) conditions and resemble the upper conducting airways, both morphologically and functionally. We validated the platform with the pathogen Mycoplasma mycoides subsp. capri strain GM12 belonging to the bacterial class of Mollicutes and with influenza D virus, a member of the Orthomyxoviridae family. These experiments highlighted the broad applicability of our platform to study caprine respiratory infections and will contribute to the reduction of the overall use of animals for in vivo models.

\section{Materials and methods}

\subsection{Establishment of caprine airway epithelial cell (AEC) cultures}

Tissue samples containing primary caprine airway cells were aseptically removed from lungs of three healthy goats slaughtered in Diemtigtal, Canton of Berne. The goats (two female and one male) belonged to the Saanen breed, a Swiss domestic goat breed, and were of a juvenile age at time of slaughter. A $10 \mathrm{~cm}$ long part of tracheobronchial tissue was resected from the bifurcation of the trachea. Isolation of the primary bronchial epithelial cells was performed as previously described and cells were cryopreserved at $-150^{\circ} \mathrm{C}$ for later use (Gultom et al., 2020).

The establishment of caprine airway epithelial cell (AEC) cultures was basically done as reported for mammalian species (Gultom et al., 2020) with a few modifications. Briefly, the cells were thawed and seeded on type I collagen-coated (Sigma) T75 flasks (TPP) in bronchial epithelial growth medium (BEGM), which was modified by adding the following supplements: HEPES (0.015 mol/L; ThermoFisher), A83-01 (1 $\mu \mathrm{mol} / \mathrm{L}$; Tocris), Y27632 (5 $\mu \mathrm{mol} / \mathrm{L}$; Abcam) and Isoprotenerol $(3 \mu \mathrm{M} / \mathrm{L}$; Tocris). After 4-6 days, when the cells reached $80 \%-90 \%$ confluence, cells were trypsinized using Gibco ${ }^{\mathrm{TM}}$ TrypLE ${ }^{\mathrm{TM}}$ Express Enzyme (1X), phenol red and seeded on Corning ${ }^{\circledR}$ Transwell ${ }^{\circledR}$ polyester membrane cell culture inserts ( $6.5 \mathrm{~mm}$ diameter, $0.4 \mu \mathrm{m}$ pore size) at a density of 2 $\times 10^{\wedge} 5$ cells per insert, maintained in BEGM. Once cells reached confluence, the apical and the basolateral medium was replaced by ALI medium supplemented with A83-01 $(1 \mu \mathrm{mol} / \mathrm{L})$ and Y27632 $(5 \mu \mathrm{mol} / \mathrm{L})$ and were kept for 2-3 more days in a Liquid-Liquid interface system. Afterwards, cells were airlifted by removing the apical medium. For cellular differentiation, ALI medium was supplemented with the following modifications: DAPT ( $5 \mu \mathrm{mol} / \mathrm{L}$; Tocris), increased working concentration of epidermal growth factor (EGF) (5.5 ng/mL; Repligen) and Hydrocortisone $(0.472 \mu \mathrm{g} / \mathrm{mL}$; Sigma). The medium was changed every 2-3 days, and the apical surface of the cell culture was washed once a week with TEER solution $\left(\mathrm{NaCl} 0.9 \%, \mathrm{CaCl}_{2} 1.25 \mathrm{mmol} / \mathrm{L}\right.$ and HEPES $10 \mathrm{mmol} / \mathrm{L}$ dissolved in distilled water; Merck KGaA). The AEC cultures were allowed to differentiate for at least four weeks at $37{ }^{\circ} \mathrm{C}$ with $5 \% \mathrm{CO}_{2}$ in a humidified atmosphere. All AEC cultures were confirmed to be free from mycoplasma cell culture contaminants using the MycoAlert ${ }^{\mathrm{TM}}$ PLUS Mycoplasma Detection Kit (Lonza).

\subsection{Histological examination}

The well-differentiated AEC cultures were washed apically with TEER solution and the medium from the basolateral compartment was removed before fixation with $4 \%$ formalin (Formafix) for $20 \mathrm{~min}$ at room temperature. Following fixation, cells were washed twice basolaterally and apically with HBSS (Gibco) after which fixed cell cultures were paraffin-embedded, stained with haematoxylin and eosin (HE) and finally sectioned according to standard methods. The samples were visualized using an EVOS FL Auto 2 imaging system (ThermoFisher), and images were processed with Fiji software package v1.53 (Schindelin et al., 2012). Tissue from an healthy goat was fixed with formalin, paraffin-embedded and stained with HE using standard methods (Liljander et al., 2019).

\subsection{Scanning electron microscopy (SEM)}

Airway epithelial cell cultures were thoroughly washed with TEER solution and fixed with $2.5 \%$ glutaraldehyde (Merck) in $0.1 \mathrm{M}$ cacodylate buffer, $\mathrm{pH} 7.4$, for $24 \mathrm{~h}$ at room temperature. Thereafter, samples were either processed for SEM immediately or stored at $4{ }^{\circ} \mathrm{C}$ until later use. Fixed AEC culture samples were washed three times in $0.1 \mathrm{M}$ cacodylate buffer $\mathrm{pH} 7.4$ and post-fixed with $1 \% \mathrm{OsO}_{4}$ (Polysciences) in $0.1 \mathrm{M}$ cacodylate buffer for $30 \mathrm{~min}$. After two washes in $0.1 \mathrm{M}$ cacodylate buffer, the samples were dehydrated through an ascending ethanol series and dried by evaporation of Hexamethyldisilazane (Merck) as described elsewhere (Stoffel et al., 2002, 1993; Stoffel and Friess, 2002). Transwell membranes containing AEC cultures were excised from the insert holder by means of a biopsy punch (Stiefel biopsy punch $6 \mathrm{~mm}$, DocCheck Shop). The specimens were mounted onto aluminium stubs (Agar Scientific Ltd.) with conductive adhesive carbon tabs (Ted Pella) and sputter-coated with approximately $15 \mathrm{~nm}$ of platinum in a high-vacuum coating unit (CCU-10, Safematic, Zizers). Secondary electron micrographs were obtained with a field emission scanning electron microscope DSM 982 Gemini (Zeiss) at an accelerating voltage of $5 \mathrm{kV}$ at a working distance of $5-8 \mathrm{~mm}$.

\subsection{Viruses and bacteria}

Virus infections were performed with influenza D virus (IDV) strain D/bovine/Oklahoma/660/2013. The propagation and the generation of the virus stock have been described elsewhere (Holwerda et al., 2019).

For this study we used the Mycoplasma mycoides subsp. capri strain GM12 (DaMassa et al., 1983) wildtype. The Mycoplasma was grown in SP5 medium (Labroussaa et al., 2016) overnight at $37{ }^{\circ} \mathrm{C}$ until they reached a pH of 6.2-6.35. The aliquots were supplemented $1: 3$ with 60 $\%$ glycerol (final concentration $15 \%$ ) and then stored at $-80{ }^{\circ} \mathrm{C}$ for further use. The number of viable mycoplasma cells was determined as colony forming units per millilitre $(\mathrm{CFU} / \mathrm{mL})$ by plating ten-fold serial dilutions on SP5 agar plates.

\subsection{Viral infection}

The differentiated caprine AEC cultures were washed once with TEER solution and then apically inoculated with 10,000 tissue culture infectious dose 50 (TCID $_{50}$ ) of IDV in a total volume of $100 \mu \mathrm{L}$ per insert. The TEER solution alone was used for mock-infected AEC cultures. The AEC cultures were incubated for $1 \mathrm{~h}$ at $37{ }^{\circ} \mathrm{C}$ in humidified atmosphere with $5 \% \mathrm{CO}_{2}$. Afterwards, the inoculum was removed via pipetting, and the surface was washed three times with $200 \mu \mathrm{L}$ of TEER solution. Subsequently, the cells were incubated as previously described. To monitor the apical release of virus, $100 \mu \mathrm{L}$ of TEER solution was added apically and incubated for $10 \mathrm{~min}$ at room temperature prior to collection. Basal release of virus was monitored by collecting $100 \mu \mathrm{L}$ of the medium at every time point. The removed volume of medium was replaced by $100 \mu \mathrm{L}$ of fresh ALI medium. All samples were stored 1:1 with virus transport medium at $-80^{\circ} \mathrm{C}$ for later analyses (Gultom et al., 2020). 


\subsection{Bacterial infection}

Three days prior to infection, the basolateral medium of the AEC cultures was changed to streptomycin-free caprine ALI medium, since mycoplasmas are sensitive to streptomycin. At the day of infection, AEC cultures were washed once with TEER and then infected with a multiplicity of infection (MOI) of 0.1 , corresponding to 20,000 colony forming units in $200 \mu \mathrm{L}$ of TEER solution. The total number of epithelial cells was determined to be approximately 200,000 cells per insert as determined by examination from multiple fields of fully differentiated caprine AEC cultures by fluorescence microscopy. For the calculation of cell numbers, two fluorescence-stained inserts per donor were used. Per insert, the number of cells in two to three different areas of around 200 $\mu \mathrm{m} \times 150 \mu \mathrm{m}$ were counted using Fiji. These numbers were used to extrapolate the number of cells for the whole insert. The infection procedure and collection of samples were performed as described above for IDV, except that at the end of the experiment, we additionally collected the cells from the membranes by trypsinization with $100 \mu \mathrm{L}$ of $\mathrm{Gibco}^{\mathrm{TM}}$ TrypLE ${ }^{\mathrm{TM}}$ Express Enzyme (1X), phenol red (30 min at $37{ }^{\circ} \mathrm{C}$ ) to assess the number of cell-bound mycoplasmas. The collected samples were directly processed to determine the relative number of mycoplasmas, measured as colour-changing units (CCU) (Stemke and Robertson, 1982) in 3 technical replicates. Colour-changing units were measured by ten-fold serial dilutions of the Mycoplasma-containing samples cultured in liquid SP5 media. A shift in colour from red to yellow through acidification indicated growth as a results of Mycoplasma metabolism. The dilutions were incubated for 7 days at $37^{\circ} \mathrm{C}$.

To determine the growth of $\mathrm{Mmc}$ in caprine ALI medium, different concentrations of $\mathrm{Mmc}$ were added to $500 \mu \mathrm{L}$ streptomycin-free caprine ALI medium. These experiments were performed in 24-well cell culture plates (Costar). At each time point, $100 \mu \mathrm{L}$ of the medium was removed and used for determination of the mycoplasma load, as described above. The removed volume of ALI medium was replaced by fresh ALI medium.

\subsection{Real-time quantitative reverse transcription polymerase chain reaction (real time $q R T-P C R$ )}

A real time qRT-PCR was performed on IDV samples as previously described (Holwerda et al., 2019). For quantification of the viral kinetics of IDV, viral RNA was extracted from $50 \mu \mathrm{L}$ of the sample using the NucleoMag VET kit for viral and bacterial RNA/DNA from veterinary samples (Macherey-Nagel AG), according to the manufacturer's guideline, on a Kingfisher Flex Purification system (ThermoFisher). Two microliters of extracted RNA were used per reaction employing the TaqMan $^{\mathrm{TM}}$ Fast Virus 1-Step Master Mix (ThermoFisher) according to the manufacturer's protocol. The sequence of the forward primer was 5'-ATGCTGAAACTGTGGAAGAATTTTG-3', and that of the reverse primer was 5'-GGTCTTCCATTTATGATTGTCAACAA-3'. The sequence of the probe was 5'-FAM-AAGGTTTATGTCCATTGTTTCA-BHQ1-3'. A standard curve of the P42-segment of IDV, cloned in pHW2000 plasmid, was included to interpolate the number of genomic equivalents (Holwerda et al., 2019). Measurements were performed on a LightCycler ${ }^{\circledR}$ 480 instrument and analyzed using the accompanied software package (Roche). The data was further processed in GraphPad Prism 8.

\subsection{Immunofluorescence}

The caprine AEC cultures were formalin-fixed and stained for immunofluorescence as described elsewhere (Gultom et al., 2020). Tight junctions were visualized with monoclonal Alexa Fluor ${ }^{\circledR}$ 594-conjugated mouse anti-ZO-1 antibody (1:100, ThermoFisher). Cilia were visualized using Alexa Fluor ${ }^{\circledR}$ 647-conjugated rabbit anti- $\beta$-tubulin antibody (1:200, Cell Signaling Technology $\left.{ }^{\circledR}\right)$. To detect IDV-positive cells, we used a custom-made rabbit polyclonal antibody directed against the nucleoprotein of the prototypic D/bovine/Oklahoma/660/2013 strain $\left(1: 200\right.$, GenScript $\left.{ }^{\circledR}\right)$. This primary antibody was detected with the Alexa Fluor ${ }^{\circledR}$ 488-conjugated donkey anti-rabbit IgG $(\mathrm{H}+\mathrm{L})$ antibody (Jackson Immunoresearch) used at a dilution of 1:800. All samples were counterstained with DAPI (4',6-diamidino-2-phenylindole; ThermoFisher) to visualize nuclei. Thereafter, the membranes of the inserts with adhering fixed cells were dissected, mounted onto slides with DakoFluorescence Mounting Medium (Agilent) and then coverslipped with ProLong ${ }^{\text {TM }}$ Diamond Antifade Mountant (ThermoFisher). Images were acquired using the EVOS ${ }^{\circledR}$ FL Auto 2 imaging system. Data analysis and processing were performed with Fiji (distribution of ImageJ) software (Schindelin et al., 2012).

\subsection{Titration of virus with $50 \%$ tissue culture infectious dose assay}

HRT-18 G cells were seeded at a concentration of 40,000 cells per well in a flat -bottom 96-well plate (TPP). After $24 \mathrm{~h}$, the medium was removed, and the cells were washed once with PBS. Fifty $\mu \mathrm{L}$ infection medium (MEM + GlutaMAX (Gibco), supplemented with $0.5 \%$ bovine serum albumin (Sigma-Aldrich), $15 \mathrm{mmol} / \mathrm{L}$ of HEPES (Gibco), $100 \mu \mathrm{g} /$ $\mathrm{mL}$ Streptomycin and 100IU/mL Penicillin (Gibco) and $0.25 \mu \mathrm{g} / \mathrm{mL}$ bovine pancreas-isolated acetylated trypsin (Sigma-Aldrich)) was added to each well. The samples of the infection experiments were diluted 1:10 with infection medium, from which $50 \mu \mathrm{L}$ were added to the HRT-18 G cells in six technical replicates. Afterwards, cells were incubated at $37{ }^{\circ} \mathrm{C}$ in a humidified incubator with $5 \% \mathrm{CO}_{2}$. After $120 \mathrm{~h}, 50 \mu \mathrm{L}$ of the supernatant were used as input for a hemagglutination assay to determine the viral titre. The viral titre was calculated according to the protocol of Spearman-Kärber (Kärber, 1931).

\subsection{Hemagglutination assay}

Chicken blood for the hemagglutination assay was obtained from SPF-bred white Leghorn chickens in compliance with the Animal Welfare Act (TSchG SR 455), the Animal Welfare Ordinance (TSchV SR 455.1), and the Animal Experimentation Ordinance (TVV SR 455.163) of Switzerland. The procedure was reviewed by the ethical committee for animal experiments of the canton of Bern and approved by the cantonal veterinary authorities (Amt für Landwirtschaft und Natur LANAT, Veterinärdienst VeD, Bern, Switzerland) with the agreements BE87/17 and BE92/20. The red blood cells were diluted 1:1 in Alsever's solution and used within three weeks. To perform the hemagglutination assay, the red blood cells were diluted to $1 \%$ in ice-cold PBS, as described previously (Holwerda et al., 2019).

\section{Results}

\subsection{Establishment of well-differentiated caprine AEC cultures}

Caprine primary airway cells originating from three different donors were used. First, the primary cells were cultured in flasks until they reached confluence, which took 5-6 days. Afterwards, cells were seeded for proliferation on permeable polyester membrane inserts in a liquidliquid interface system for 6-7 days. The proliferation was monitored by light microscopy. After reaching confluence, which was confirmed via absence of leakage of basolateral medium into the apical compartment, the cells were apically exposed to air. After exposure to ALI conditions, the cell cultures well-differentiated within 7-10 days. We adapted the ALI medium to caprine cells via several rounds of testing different ALI medium formulations (Table 1). The differentiation was confirmed by the presence of different cell types (secreting-, basal- and ciliated cells), ciliary activity, mucus production and pseudostratification. The ciliary activity was examined and confirmed by light microscopy. Three to 5 days after exposure to ALI conditions ciliary activity first appeared at the borders of the insert but was also observed in the centre of the insert 4-5 days later. The presence of cilia was corroborated by scanning electron microscopy and immunofluorescence microscopy (Fig. 1). Moreover, immunofluorescence staining of tight 
Table 1

Effect of different compositions of ALI media towards caprine AEC cultures.

\begin{tabular}{lllll}
\hline $\begin{array}{l}\text { Parameter } \\
\text { tested }\end{array}$ & $\begin{array}{l}\mathrm{HC} \\
(\mu \mathrm{g} / \\
\mathrm{mL})\end{array}$ & $\begin{array}{l}\mathrm{EGF} \\
(\mu \mathrm{g} / \\
\mathrm{mL})\end{array}$ & $\begin{array}{l}\text { DAPT } \\
(\mu \mathrm{M})\end{array}$ & Results \\
\hline Condition 1 & 0.472 & 5.5 & 5 & $\begin{array}{l}\text { Well-differentiated caprine AEC } \\
\text { Condition 2 }\end{array}$ \\
0.472 & 0.5 & 10 & $\begin{array}{l}\text { Small percentage of ciliated cells } \\
\text { Compared to Condition 1: less } \\
\text { ciliated cells, wide variation in cell } \\
\text { size }\end{array}$ \\
\hline
\end{tabular}

HC: Hydrocortison, EGF: Epidermal growth factor, DAPT: N-[N-(3,5-Difluorphenacetyl)-L-alanyl]-S-phenylglycin-tert-butylester.

junctions to visualize cell borders and cilia revealed the presence of both, ciliated and non-ciliated cells. Mucus production was observed after 6-8 days exposure to ALI conditions. Mucus was visible by eye or light microscopy. To prevent accumulation of mucus, the cells were washed once a week. We were able to maintain the AEC cultures up to maximal 2 months in the ALI system. Afterwards, the ciliary activity decreased, and the cultures lost their confluence as a consequence of cell death. All three donors reached differentiation in the same time range and showed comparable results. Histologically, the AEC cultures showed features observed in tissue samples from healthy goats (Fig. S1).

\subsection{Infectivity, replication and cell tropism of IDV}

Well-differentiated caprine AECs of three different donors were apically inoculated with 10,000 TCID $_{50}$ IDV for one hour. Afterwards the cell cultures were washed 3 times to remove non-adherent viruses, and incubation was continued. To confirm that caprine AECs are susceptible to IDV, we visualized the IDV-Nucleoprotein (IDV-NP) by fluorescence microscopy after $96 \mathrm{~h}$ of infection (Fig. 2). As expected, only AECs infected with IDV showed the presence of IDV-NP. This demonstrates that the caprine AEC cultures are susceptible to IDV adherence and replication.

As AECs encompass different cell types, we wanted to examine if IDV had a tropism to a certain cell type. At $96 \mathrm{~h}$ post infection, we visualized tight junctions and cilia by fluorescence microscopy using the anti-ZO-1 and the anti- $\beta$-tubulin antibodies, respectively. This experiment revealed that IDV-NP positive cells overlap with $\beta$-tubulin positive areas. Therefore, we concluded that IDV preferably infects and replicates within ciliated cells. Nevertheless, the target cell tropism is not strictly limited to ciliated cells as we also found non-ciliated IDV-NP positive cells occasionally (data not shown).

To investigate the replication kinetics, apical washes and basolateral medium were collected at 24, 48, 72 and $96 \mathrm{~h}$ post infection. The samples were analysed for virus progeny by quantitative real-time reverse transcription PCR (Fig. 3A, B). The results showed that the apical release of viral RNA increased over time with small differences between the donors. Additionally, viral RNA was detected in the basolateral medium, however in lower quantity. Therefore, we concluded that the viral shedding mainly occurs at the apical membrane.

Furthermore, we examined the virus titres employing a hemagglutination assay to confirm the release of viral particles (Fig. 3C, D). An increase of viral particles in the apical washes over time was observed for all samples analysed. In contrast, virus was only detected in the basolateral medium for one donor animal at $72 \mathrm{~h}$ post infection. These results support a clear correlation between the quantity of viral RNA and the virus titre. In summary, caprine AECs provide the necessary molecular requirements to enable an efficient replication of infectious IDV.

\subsection{Growth of Mmc on caprine AEC}

To investigate adhesion and growth of the caprine pathogen $\mathrm{Mmc}$ GM12 on caprine AEC cultures, we followed the same experimental setup as for IDV with some adaptations. Well-differentiated caprine AECs derived from three different donors were inoculated with GM12 at a MOI of 0.1 for $1 \mathrm{~h}$ at $37^{\circ} \mathrm{C}$. We also tested MOI 1 and MOI 10 (Fig. S2), but for the final experiments we chose an MOI of 0.1 as it likely mimics a more natural pathogen load upon infection. Afterwards, non-attached bacteria were removed by washing the cultures three times. At 24,48 , 72 and $96 \mathrm{~h}$ post infection, apical washes were collected to quantify non-

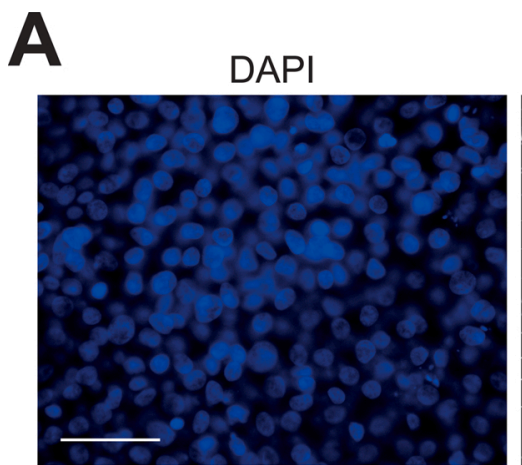

ß-Tubulin IV

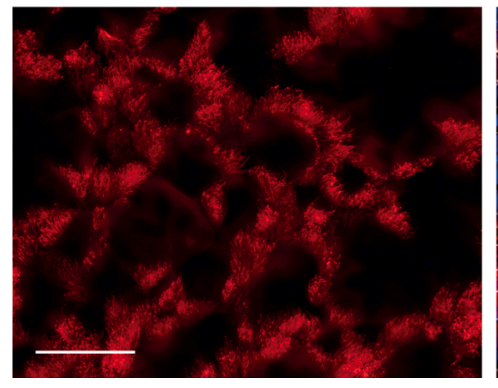

$\mathrm{ZO}-1$

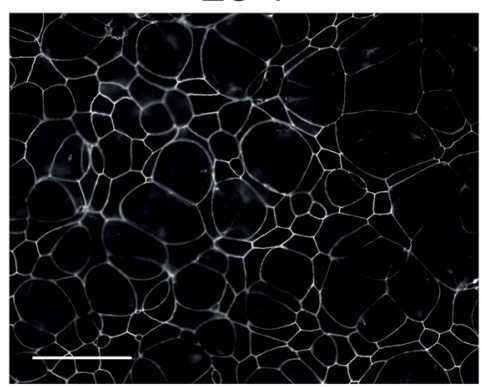

Merge

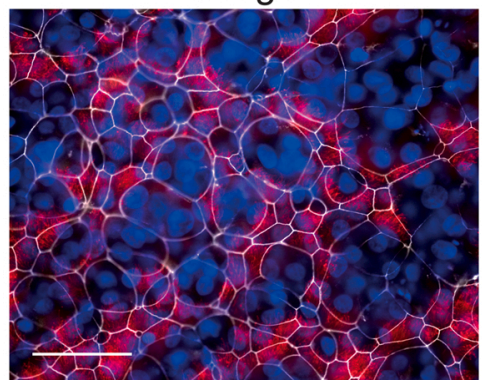

B
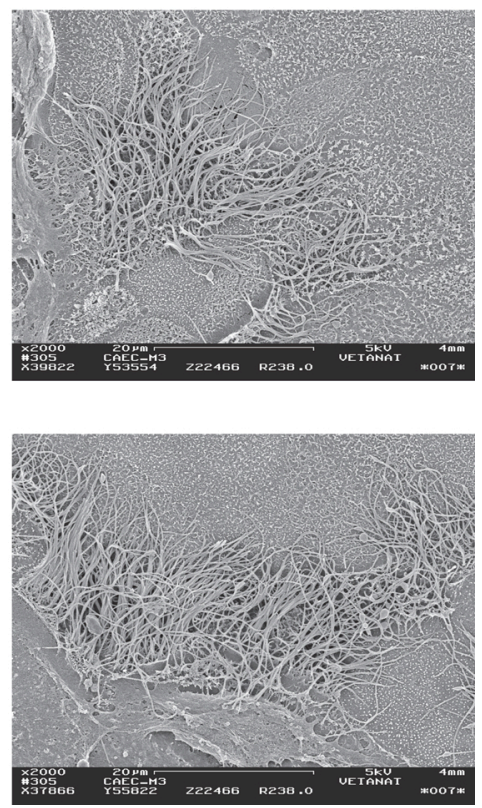

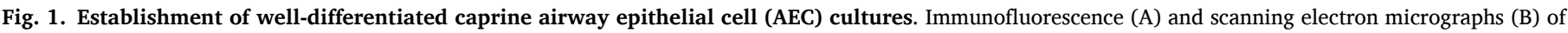

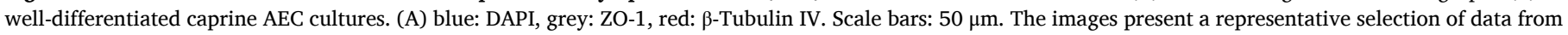

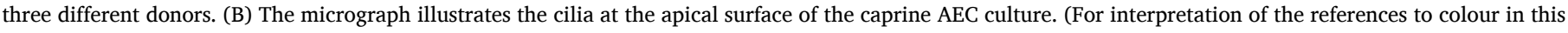
figure legend, the reader is referred to the web version of this article). 
A

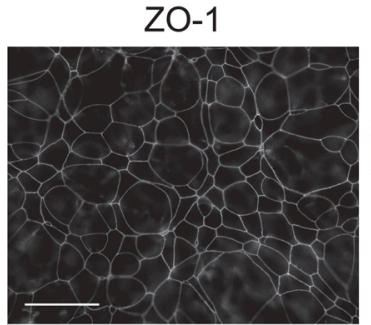

IDV-NP

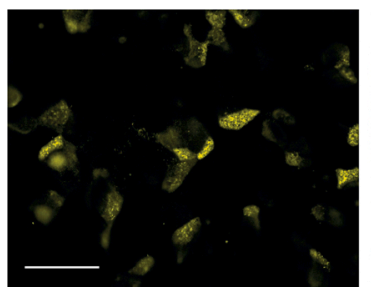

B

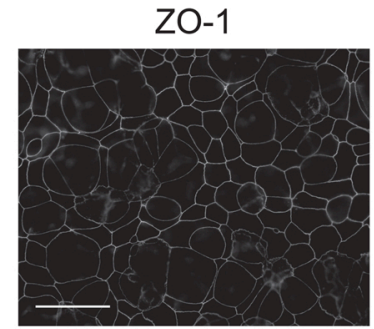

IDV-NP

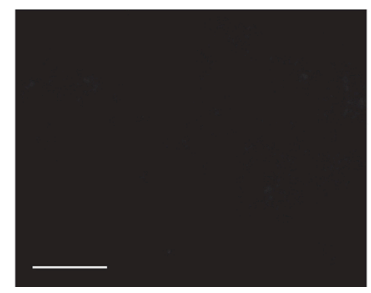

ß-Tubulin IV

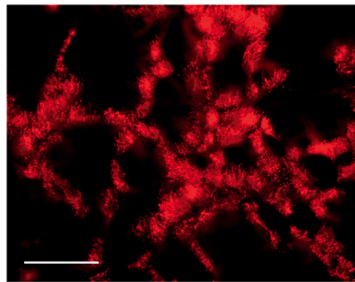

Merge

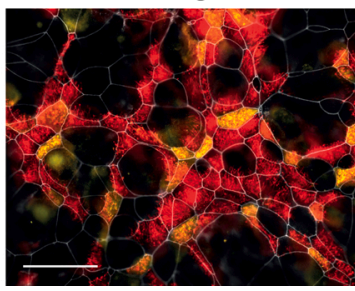

Mock-infected

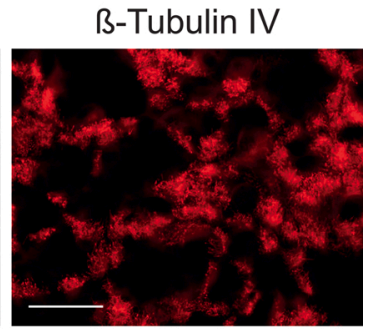

Merge

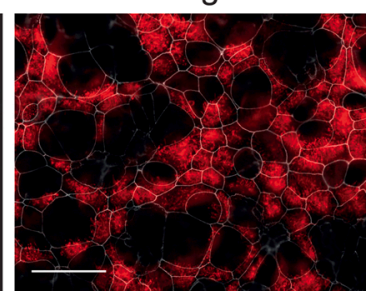

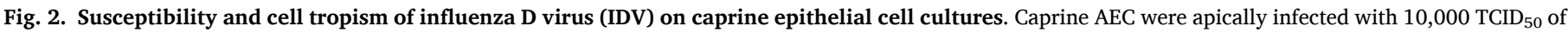

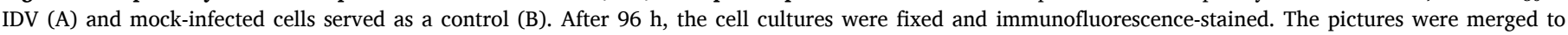

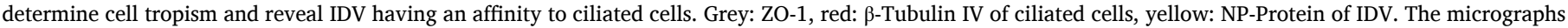

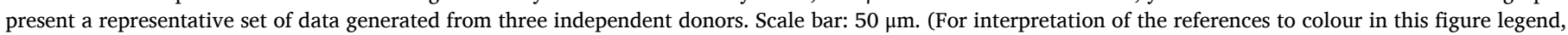
the reader is referred to the web version of this article).
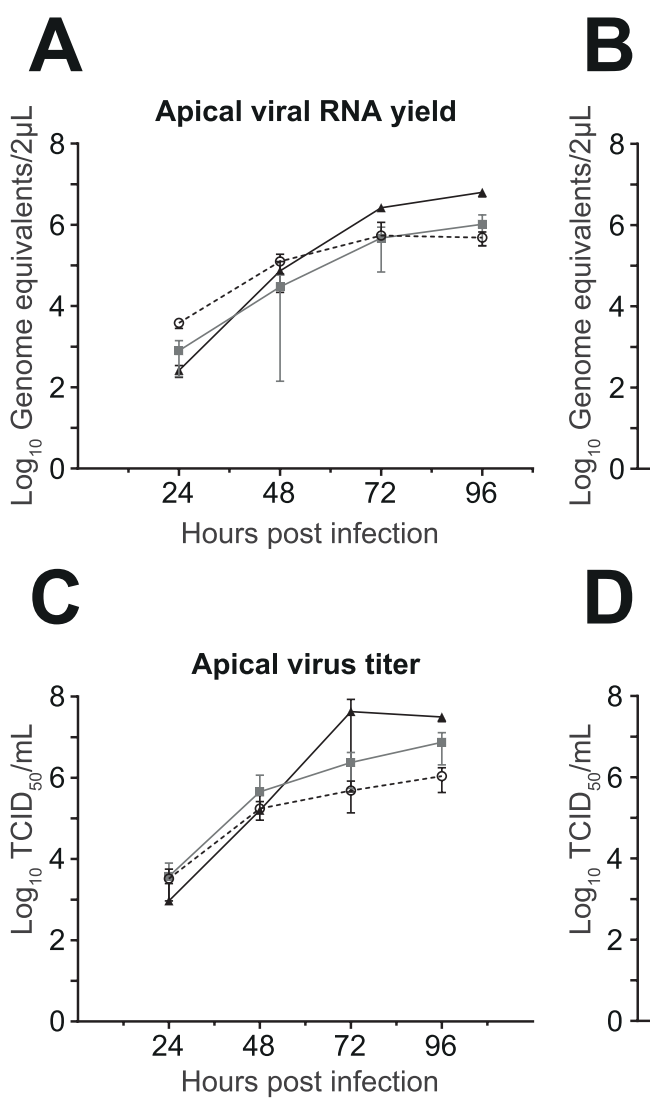



$D$

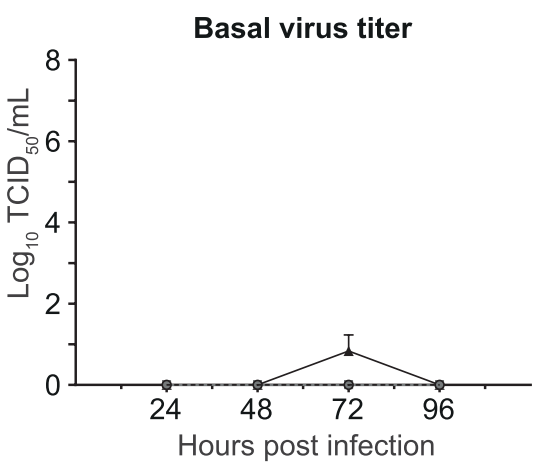

Fig. 3. Replication of influenza D virus (IDV) on welldifferentiated caprine airway epithelial cell (AEC) cultures. Caprine AEC cultures were apically inoculated with $10,000 \mathrm{TCID}_{50}$ of Influenza virus D. Apical wash (A\&C) and basal medium (B\&D), respectively, were collected at different time points post inoculation (x-axis). The monitored viral RNA yield (A\&B) is given as genome equivalent per $2 \mu \mathrm{L}$ of isolated RNA (y-axis). Viral Titer (C\&D) is given as $\mathrm{TCID}_{50} / \mathrm{mL}$ and was measured by hemagglutination assay. The results are displayed as means and standard deviation (SD) from triplicates from three individual biological donors. Each symbol represents one donor (black dotted line with circle: female donor 1, grey line with square: female donor 2, black line with triangle: male donor). Mock infected cultures used as control were all negative. adherent live mycoplasmas. Moreover, after the final wash at $96 \mathrm{~h}$ post infection, the AECs were also removed from their support via trypsin treatment to quantify the number of mycoplasmas intimately attached to the cells. The trypsin treatment did not affect the viability of the mycoplasmas (Fig. S3). Serial dilutions were made from all samples to quantify live mycoplasmas by measuring CCUs as described elsewhere
(Jores et al. 2019).

A comparable number of mycoplasma cells $\left(\sim 10^{7} \mathrm{CCU} / \mathrm{mL}\right)$ were observed in AECs derived from all donors at 72 and $96 \mathrm{~h}$ post infection (Fig. 4A). This demonstrates that AECs cultured under ALI conditions deliver all the nutrients required to support colonization of $\mathrm{Mmc}$. The number of mycoplasmas intimately attached to the AECs is similar to the 
A



B

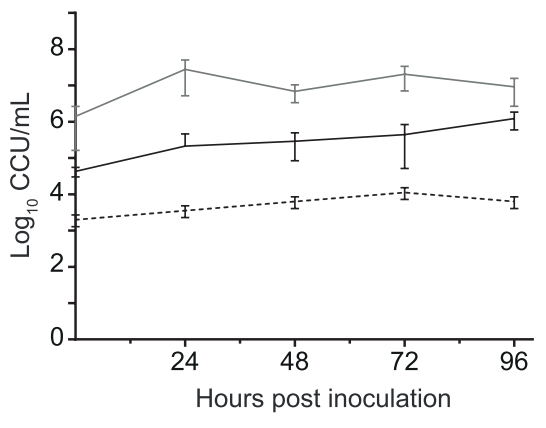

Fig. 4. Replication of $M$. mycoides subsp. capri (Mmc) GM12 on caprine AEC culture and in ALI medium. (A) Welldifferentiated caprine AEC cultures were apically inoculated with $\mathrm{Mmc}$ at an MOI of 0.1. Non-adherent bacteria in apical washes were determined via colour changing units $\mathrm{CCU} / \mathrm{mL}$ (yaxis) at indicated timepoints (x-axis). After $96 \mathrm{~h}$, the cells were removed from the inserts and adherent bacteria were counted (grey box on the right). The results are displayed as means and standard deviation (SD) from triplicates from three individual biological donors: black dotted line with circle: female donor 1 , grey line with square: female donor 2, black line with triangle: male donor. Mock-infected cultures used as controls were negative. (B) Caprine-adapted ALI medium was inoculated with three different concentrations of $\mathrm{Mmc}$. The number of $M m c$ was measured with $\mathrm{CCU} / \mathrm{mL}$ (y-axis) at indicated timepoints (x-axis). The results are displayed as means and standard deviation (SD) from triplicates.

number of mycoplasmas collected after washes, which points towards the presence of mycoplasmas with different avidity towards the AECs. We did not observe major morphological changes after infection. To prove that the growth of $\mathrm{Mmc}$ described above is associated with AECs, we assessed the persistence and growth of $\mathrm{Mmc}$ in streptomycin-free caprine ALI medium using 3 different bacterial concentrations. Fig. 4B shows only a marginal increase of $\mathrm{Mmc}$ over $96 \mathrm{~h}$ incubated in ALI medium. This confirms that mycoplasmas survive in ALI medium but were not able to replicate efficiently. In summary, the experiments confirmed that caprine mycoplasmas can be studied with respect to adhesion using caprine AEC cultures grown under ALI.

\section{Discussion}

Caprine respiratory infections were most often studied on caprine and non-caprine, primary or immortalized cells grown in submersion cell cultures (Aye et al., 2015; Besozzi et al., 2019; Lanave et al., 2019; Sun et al., 2021). All cell lines used to study respiratory infections have their own limitations in terms of offereing only one cell type that is submerged in cell culture medium, while the respiratory tissue consists of many different cell types most of which contribute to an air-tissue interface. To overcome this limitation, we established a caprine in vitro model based on well-differentiated AECs grown in an ALI system. Such in vitro systems have been first developed to study human infections (Whitcutt et al., 1988), were later adapted to ruminant species such as sheep (O'Boyle et al., 2017) and cattle (Cozens et al., 2018), but have not been reported for goats to the best of our knowledge. Characteristics of differentiated AECs such as the presence of ciliated and non-ciliated cells, ciliary activity and mucus production, reported for bovine and ovine AECs (Cozens et al., 2018; O'Boyle et al., 2018), were also observed in our caprine in vitro model. The development of a differentiated AEC model of the caprine respiratory tract requires the optimization of the growth medium towards a defined medium that supports the differentiation of the caprine AECs. Here, we present a standardised medium with defined concentrations of growth factors. The improvement of the ALI medium via testing different formulations of key growth factors such as EGF, retinoic acid (RA) and triiodothyronine (T3) was key in the setup of our AEC model. Our medium differs from the medium used in the setup of bovine AECs in the amount of EGF, DAPT and slightly in the amount of hydrocortisone. Future research efforts towards optimization of the ALI medium reported here are likely to benefit the caprine AEC culture model and it would also increase our knowledge on key growth factors influencing the response of caprine epithelia. The model presented here mirrors an environment present in the in vivo situation, but lacking cells of the immune system. It has the potential to gain widespread use to study host-pathogen interactions with focus on responses on the different cell types provided by the AEC cultures and to foster the 3R (Replace, Reduce, Refine) principles.

We selected the viral pathogen IDV for probing our in vitro platform.
IDV belongs to the relatively new genus Influenzavirus $D$ within the Orthomyxoviridae family. It was first isolated in 2011 from swine with flu-like symptoms (Hause et al., 2014). IDV has a large host range, as serological signatures were detected in horses, camels and small ruminants. More recently, the IDV carriage of goats was confirmed using RT-PCR in China (Zhai et al., 2017). These data indicated that goats are a reservoir, hence IDV is able to replicate in the animal's upper respiratory tract, which was tested in our study. Our results confirm not only that IDV has a tropism for ciliated cells but also that it can efficiently replicate on caprine AEC.

Since persistent Mycoplasma pneumoniae infections have been modelled using human AECs (Prince et al., 2018), we assessed whether our in vitro system can be used to monitor colonization of caprine mycoplasmas. Therefore, we established an infection protocol for Mycoplasma mycoides subsp. capri, a highly virulent pathogen for goats. It causes the MAKePS syndrome, which can present as Mastitis, Arthritis, Keratitis, Pneumonia or Septicaemia (Thiaucourt and Bolske, 1996). We used the strain GM12, which was isolated from an outbreak associated with high mortality in the USA (DaMassa et al., 1983) and has been used to experimentally infect goats transtracheally (Jores et al., 2019a, b). Our results confirm the capacity of Mmc GM12 to colonize caprine AEC cultures in a manner that closely mimicks the situation in vivo.

\section{Conclusion}

Here we established for the first time well-differentiated caprine AEC cultures grown under ALI conditions. Moreover, we developed infection protocols for IDV and $\mathrm{Mmc}$ suitable to study host-pathogen interactions using the caprine AEC cultures. It thus provides an in vitro experimental platform to study caprine respiratory infections, which is in line with the 3R principles and the animal welfare act. A better understanding of hostpathogen interaction in the course of caprine respiratory infections will foster the development of rational vaccines or treatments. Caprine AEC cultures complement other existing caprine in vitro and ex vivo platforms to foster our knowledge on respiratory infections in goats.

\section{Author contributions}

JJ \& VT designed the study. MS, JJ and VT drafted the manuscript. MS, LL, MG, PV, SCP, NE, FL and RD developed the model and performed the infections. MHS did the electron micrographs and ACB did the histology. All authors read and approved the final manuscript.

\section{Declaration of Competing Interest}

The authors declare that they have no known competing financial interests or personal relationships that could have appeared to influence the work reported in this paper. 


\section{Acknowledgements}

This work was funded by the University of Bern and the Institute of Virology and Immunology (IVI). Joerg Jores and Fabien Labroussaa were additionally supported by International Development Research Centre (Grant ID: 108625). We thank Helga Mogel from the Division of Veterinary Anatomy for support with the Scanning Electron Microscopy. Microscopy was performed on equipment supported by the Microscopy Imaging Center (MIC) at the University of Bern.

\section{Appendix A. Supplementary data}

Supplementary material related to this article can be found, in the online version, at doi:https://doi.org/10.1016/j.vetmic.2021.109067.

\section{References}

Aye, R., Mwirigi, M.K., Frey, J., Pilo, P., Jores, J., Naessens, J., 2015. Cyto-adherence of Mycoplasma mycoides subsp. mycoides to bovine lung epithelial cells. BMC Vet. Res. $11,27$.

Banyard, A.C., Parida, S., Batten, C., Oura, C., Kwiatek, O., Libeau, G., 2010. Global distribution of peste des petits ruminants virus and prospects for improved diagnosis and control. J. Gen. Virol. 91, 2885-2897.

Besozzi, M., Lauzi, S., Lelli, D., Lavazza, A., Chiapponi, C., Pisoni, G., Vigano, R., Lanfranchi, P., Luzzago, C., 2019. Host range of mammalian orthoreovirus type 3 widening to alpine chamois. Vet. Microbiol. 230, 72-77.

Chakraborty, S., Kumar, A., Tiwari, R., Rahal, A., Malik, Y., Dhama, K., Pal, A., Prasad, M., 2014. Advances in diagnosis of respiratory diseases of small ruminants. Vet. Med. Int. 2014, 508304.

Cozens, D., Grahame, E., Sutherland, E., Taylor, G., Berry, C.C., Davies, R.L., 2018. Development and optimization of a differentiated airway epithelial cell model of the bovine respiratory tract. Sci. Rep. 8, 853.

DaMassa, A.J., Brooks, D.L., Adler, H.E., 1983. Caprine mycoplasmosis: widespread infection in goats with Mycoplasma mycoides subsp mycoides (large-colony type). Am. J. Vet. Res. 44, 322-325.

FAO, 2020. Food and Agriculture Organization of the United Nations Statistical Databases. FAO. http://faostat.fao.org/.

Gultom, M., Laloli, L., Dijkman, R., 2020. Well-differentiated primary mammalian airway epithelial cell cultures. Methods Mol. Biol. 2203, 119-134.

Hause, B.M., Collin, E.A., Liu, R., Huang, B., Sheng, Z., Lu, W., Wang, D., Nelson, E.A., Li, F., 2014. Characterization of a novel influenza virus in cattle and Swine: proposal for a new genus in the Orthomyxoviridae family. mBio 5, e00031-00014.

Holwerda, M., Kelly, J., Laloli, L., Sturmer, I., Portmann, J., Stalder, H., Dijkman, R., 2019. Determining the replication kinetics and cellular tropism of influenza d virus on primary well-differentiated human airway epithelial cells. Viruses 11.

Jores, J., Ma, L., Ssajjakambwe, P., Schieck, E., Liljander, A., Chandran, S., Stoffel, M.H., Cippa, V., Arfi, Y., Assad-Garcia, N., Falquet, L., Sirand-Pugnet, P., Blanchard, A., Lartigue, C., Posthaus, H., Labroussaa, F., Vashee, S., 2019a. Removal of a subset of non-essential genes fully attenuates a highly virulent Mycoplasma strain. Front. Microbiol. 10, 664.

Jores, J., Schieck, E., Liljander, A., Sacchini, F., Posthaus, H., Lartigue, C., Blanchard, A., Labroussaa, F., Vashee, S., 2019b. In vivo role of capsular polysaccharide in Mycoplasma mycoides. J. Infect. Dis. 219, 1559-1563.

Jores, J., Baldwin, C., Blanchard, A., Browning, G.F., Colston, A., Gerdts, V., Goovaerts, D., Heller, M., Juleff, N., Labroussaa, F., Liljander, A., Muuka, G., Nene, V., Nir-Paz, R., Sacchini, F., Summerfield, A., Thiaucourt, F., Unger, H.,
Vashee, S., Wang, X., Salt, J., 2020. Contagious Bovine and Caprine Pleuropneumonia: a research community's recommendations for the development of better vaccines. NPJ Vaccines 5, 66.

Kärber, G., 1931. Beitrag zur kollektiven Behandlung pharmakologischer Reihenversuche. Naunyn-Schmiedebergs Archiv für experimentelle Pathologie und Pharmakologie 162, 480-483.

Labroussaa, F., Lebaudy, A., Baby, V., Gourgues, G., Matteau, D., Vashee, S., SirandPugnet, P., Rodrigue, S., Lartigue, C., 2016. Impact of donor-recipient phylogenetic distance on bacterial genome transplantation. Nucleic Acids Res. 44, 8501-8511.

Lanave, G., Lucente, M.S., Siciliano, P., Zizzadoro, C., Trerotoli, P., Martella, V., Buonavoglia, C., Tempesta, M., Camero, M., 2019. Antiviral activity of PHA767491 on Caprine alphaherpesvirus 1 in vitro. Res. Vet. Sci. 126, 113-117.

Liljander, A., Sacchini, F., Stoffel, M.H., Schieck, E., Stokar-Regenscheit, N., Labroussaa, F., Heller, M., Salt, J., Frey, J., Falquet, L., Goovaerts, D., Jores, J., 2019. Reproduction of contagious caprine pleuropneumonia reveals the ability of convalescent sera to reduce hydrogen peroxide production in vitro. Vet. Res. 50, 10.

Miller, B.A., Lu, C.D., 2019. Current status of global dairy goat production: an overview. Asian-Australas J. Anim. Sci. 32, 1219-1232.

O'Boyle, N., Sutherland, E., Berry, C.C., Davies, R.L., 2017. Temporal dynamics of ovine airway epithelial cell differentiation at an air-liquid interface. PLoS One 12, e0181583.

O'Boyle, N., Sutherland, E., Berry, C.C., Davies, R.L., 2018. Optimisation of growth conditions for ovine airway epithelial cell differentiation at an air-liquid interface. PLoS One 13, e0193998.

Prince, O.A., Krunkosky, T.M., Sheppard, E.S., Krause, D.C., 2018. Modelling persistent Mycoplasma pneumoniae infection of human airway epithelium. Cell. Microbiol. 20.

Rosenberger, C.M., Podyminogin, R.L., Askovich, P.S., Navarro, G., Kaiser, S.M., Sanders, C.J., McClaren, J.L., Tam, V.C., Dash, P., Noonan, J.G., Jones, B.G., Surman, S.L., Peschon, J.J., Diercks, A.H., Hurwitz, J.L., Doherty, P.C., Thomas, P.G., Aderem, A., 2014. Characterization of innate responses to influenza virus infection in a novel lung type I epithelial cell model. J. Gen. Virol. 95, 350-362.

Schindelin, J., Arganda-Carreras, I., Frise, E., Kaynig, V., Longair, M., Pietzsch, T., Preibisch, S., Rueden, C., Saalfeld, S., Schmid, B., Tinevez, J.Y., White, D.J., Hartenstein, V., Eliceiri, K., Tomancak, P., Cardona, A., 2012. Fiji: an open-source platform for biological-image analysis. Nat. Methods 9, 676-682.

Stemke, G.W., Robertson, J.A., 1982. Comparison of two methods for enumeration of mycoplasmas. J. Clin. Microbiol. 16, 959-961.

Stoffel, M.H., Friess, A.E., 2002. Demonstration and cytochemical analysis of anionic sites on ejaculated boar spermatozoa: a scanning electron microscopy study using cationised colloidal gold. Histochem. Cell Biol. 117, 61-67.

Stoffel, M.H., Frethem, C., Hamilton, D.W., Friess, A.E., 1993. Improved preservation of rat epididymal sperm for high-resolution low-voltage scanning electron microscopy (HR-LVSEM). Mol. Reprod. Dev. 34, 175-182.

Stoffel, M.H., Busato, A., Friess, A.E., 2002. Density and distribution of anionic sites on boar ejaculated and epididymal spermatozoa. Histochem. Cell Biol. 117, 441-445.

Sun, M., Li, W., Zhang, W., Yang, L., Hao, F., Li, J., Mao, L., Jiang, J., Liu, M., 2021. Screening interferon antagonists from accessory proteins encoded by $\mathrm{P}$ gene for immune escape of Caprine parainfluenza virus 3. Vet. Microbiol. 254, 108980.

Thiaucourt, F., Bolske, G., 1996. Contagious caprine pleuropneumonia and other pulmonary mycoplasmoses of sheep and goats. Revue scientifique et technique 15, 1397-1414.

Weldearegay, Y.B., Muller, S., Hanske, J., Schulze, A., Kostka, A., Ruger, N., HewickerTrautwein, M., Brehm, R., Valentin-Weigand, P., Kammerer, R., Jores, J., Meens, J., 2019. Host-pathogen interactions of Mycoplasma mycoides in caprine and bovine Precision-Cut Lung Slices (PCLS) models. Pathogens 8.

Whitcutt, M.J., Adler, K.B., Wu, R., 1988. A biphasic chamber system for maintaining polarity of differentiation of cultured respiratory tract epithelial cells. In Vitro Cell. Dev. Biol. 24, 420-428.

Zhai, S.L., Zhang, H., Chen, S.N., Zhou, X., Lin, T., Liu, R., Lv, D.H., Wen, X.H., Wei, W.K., Wang, D., Li, F., 2017. Influenza d virus in animal species in Guangdong Province, Southern China. Emerg Infect Dis 23, 1392-1396. 